DOI 10.35381/cm.v7i1.465

\title{
Incubación artificial y producción de huevos
}

\section{Artificial incubation and egg production}

\author{
Diego Armando Masaquiza-Moposita \\ dmasaquiza@espoch.edu.ec \\ Escuela Superior Politécnica de Chimborazo, Riobamba \\ Ecuador. \\ https://orcid.org/0000-0001-5176-8261 \\ Junior Stalin Vargas-Hidalgo \\ junior.vargas@espoch.edu.ec \\ Escuela Superior Politécnica de Chimborazo, Riobamba \\ Ecuador \\ https://orcid.org/0000-0003-1245-7609 \\ Nelson Rene Ortiz-Naveda \\ nelson.ortiz@espoch.edu.ec \\ Escuela Superior Politécnica de Chimborazo, Riobamba \\ Ecuador. \\ https://orcid.org/0000-0003-1083-8475
}

Recibido: 30 de abril de 2021 Aprobado: 15 de julio de 2021 


\title{
RESUMEN
}

La investigación tiene por objetivo analizar que los huevos clasificados como "no aptos" (por su peso y forma), procedentes de reproductoras pesadas, pueden ser utilizados en la incubación artificial, obteniendo de ellos, pollitos aceptables para la cría de ceba. La recolección de huevos se generó en las haciendas : "AMALIA", "EL GUAYABAL" y "FLOR CANELA", ubicadas en la parroquia Madre Tierra, cantón Mera, provincia de Pastaza. El proceso de incubación en la planta "INCUPASTAZA", ubicada en la vía Shell-Madre Tierra, km 4, parroquia Madre Tierra, cantón Mera, provincia de Pastaza, Ecuador. Siendo los pollitos procedentes de los huevos grandes los que obtuvieron los mayores pesos. Sin embargo, coincidiendo con los planteamientos anteriores los pollitos de huevos pequeños no llegan a alcanzar el peso requerido, con un peso promedio de 35,14 $\mathrm{g}$, por lo que se consideran pollos no viables y también como pollitos de segunda.

Descriptores: Producción alimentaria; industria alimentaria; agroindustria. (Palabras tomadas del Tesauro UNESCO).

\begin{abstract}
The objective of the research is to analyze that the eggs classified as "not suitable" (due to their weight and shape), coming from broiler breeders, can be used in artificial incubation, obtaining from them acceptable chicks for fattening. The collection of eggs was generated in the farms: "AMALIA", "EL GUAYABAL" and "FLOR CANELA", located in the Madre Tierra parish, Mera canton, Pastaza province. The incubation process at the "INCUPASTAZA" plant, located on the Shell-Madre Tierra road, km 4, Madre Tierra parish, Mera canton, Pastaza province, Ecuador. Being the chicks from the large eggs the ones that obtained the highest weights. However, coinciding with the previous approaches, the chicks with small eggs do not reach the required weight, with an average weight of $35.14 \mathrm{~g}$, which is why they are considered non-viable chicks and also as secondclass chicks.
\end{abstract}

Descriptors: Food production; food industry; agroindustry. (Words taken from the UNESCO Thesaurus). 


\section{INTRODUCCIÓN}

La incubación es el acto por el que los animales ovíparos (sobre todo las aves) empollan o incuban los huevos sentándose sobre ellos para mantenerlos calientes y así se puedan desarrollar los embriones. (De Marchi et al 2008). De acuerdo con las informaciones de Juárez (2006) el hombre está interesado en los huevos desde tiempos inmemorables, puesto que sus requerimientos básicos incluyen la necesidad de proteínas, vitaminas y minerales y constituye un alimento recomendable para todas las edades, muy adecuado en las etapas de crecimiento y necesidades fisiológicas especiales (embarazo y lactancia) y en la dieta de personas mayores. Hoy en día, el huevo constituye aproximadamente el $30 \%$ de la proteína animal que se consume en el mundo y es evidente, que las aves serán la principal fuente de carne en el próximo siglo.

Aganga et al (2003) señalaron que en 1995 la población ganadera estática en África, que incluían las aves eran las más numerosas especies de animales de granja (más del 80 por ciento) y contribuían sustancialmente a la producción anual de huevos y carne. Urrutia (2000) expresa que se están observando los avances en la avicultura de los últimos 50 años, es difícil no entusiasmarse pensando en cómo serán los próximos 50 . Y ahí estaremos todos juntos desde la incubación hasta el matadero y el mercadeo.

Considerando los criterios de Lesson (2004), en la actualidad, la industria avícola está enfrascada en obtener linajes con alta productividad. En el caso de la producción de huevo, se presta atención al número, tamaño, calidad del cascarón del huevo y consumo de alimento, lo que avala a la industria de la reproducción, a contar cada vez con menos opciones de linaje.

Lacy (2000) asegura que esta industria ha enfrentado muchos obstáculos en el pasado, pero el futuro ciertamente presentará desafíos y oportunidades aún mayores. Una de las fuerzas significativas de la industria de pollos de ceba, ha sido su avidez para adoptar nuevas tecnologías; quizás más que cualquier otra área del sector agropecuario, ha estado dispuesta a probar y adoptar nuevas ideas, para que el productor de esta línea en 


\section{CIENCIAMATRIA}

Revista Interdisciplinaria de Humanidades, Educación, Ciencia y Tecnología

Año VII. Vol. VII. N¹. Edición Especial. 2021

Hecho el depósito de ley: pp201602FA4721

ISSN-L: 2542-3029; ISSN: 2610-802X

Universidad Nacional Experimental Francisco de Miranda (UNEFM). Santa Ana de Coro. Venezuela

Diego Armando Masaquiza-Moposita; Junior Stalin Vargas-Hidalgo; Nelson Rene Ortiz-Naveda

el siglo XXI continúe siendo competitivo. Se ha optado por nuevos métodos y equipos que reducen la mano de obra, aumentan la eficiencia, mejoran y refuerzan consistentemente los rendimientos productivos, esto los lleva a ser aún más importantes que en el pasado. Estos aspectos que se describen para la producción de pollos de ceba pueden extrapolarse a la producción de huevos con el fin de mejorar la eficiencia en la explotación de este propósito.

Por su parte, Guerra y Cabrera (2003) reconocen que uno de los elementos en que los avicultores, en su gran mayoría coinciden, es que, en ambas tecnologías los resultados no sólo se logran con un buen trabajo en las etapas finales (ceba y ponedoras comerciales) que son fiables, hay que lograr reemplazos en cantidad y calidad que aseguren las necesidades de estas producciones terminales. Es por ello que, en la actualidad, gastan grandes recursos y esfuerzos en etapas intermedias, como son la incubación artificial y los reproductores, donde se obtienen los animales para las crianzas finales como los pollos de ceba y ponedoras comerciales.

La crianza de reproductores debe garantizar los huevos fértiles y aptos necesarios para que, después del proceso de incubación, se obtengan los pollitos de primera que puedan remplazar los animales de la etapa final. Esto implica que en las etapas intermedias el manejo de los huevos y animales debe ser esmerado y óptimo, pues sobre la fertilidad de los huevos actúa un gran número de factores que son manejados por el hombre, y que de ellos depende la eficiencia y rentabilidad del proceso productivo en general, otro tanto ocurre con la incubación. Cuando estas dos etapas no son eficientes y rentables, provocan, por lo general, un aumento en el costo de las producciones finales, afectando la economía de estas etapas, o por el contrario, que no se produzcan los huevos y carne que demanda el consumo humano (Guerra 2006).

Diferentes autores, Ortega y Requejo (2000) y Summers (2004), hacen hincapié en que ningún alimento puede ser catalogado como "bueno" o "malo" en sí mismo, y sólo las dietas globales se pueden juzgar. El estudio del huevo merece una atención especial 


\section{CIENCIAMATRIA}

Revista Interdisciplinaria de Humanidades, Educación, Ciencia y Tecnología

Año VII. Vol. VII. N¹. Edición Especial. 2021

Hecho el depósito de ley: pp201602FA4721

ISSN-L: 2542-3029; ISSN: 2610-802X

Universidad Nacional Experimental Francisco de Miranda (UNEFM). Santa Ana de Coro. Venezuela

Diego Armando Masaquiza-Moposita; Junior Stalin Vargas-Hidalgo; Nelson Rene Ortiz-Naveda

debido a que, en los últimos años, este alimento se ha observado con recelo y temido por su contenido en colesterol, lo que contribuye a disminuir su presencia en la dieta media, sin embargo, no cambia su composición y mantiene estable su valor nutritivo.

El huevo de ave embrionado es un sector atractivo para aplicar nuevas tecnologías por varias razones: es inmóvil y de fácil acceso por equipos de alta automatización, entre otras más (Ricks et al. 2003).

Salazar (2008) sostiene que la incubación comercial ha avanzado mucho durante los últimos 30 años. Existen adelantos significativos en muchas áreas de producción con incrementos en la productividad de las gallinas reproductoras, en mejora de las condiciones de alojamiento y de los nidos, en la fertilidad y en el tamaño de los huevos incubados. Las incubadoras y las nacedoras, se han tornado más sofisticadas y más grandes en capacidad, y también han incrementado en forma pareja, la cantidad de huevos incubados en cada máquina.

La Incubabilidad es el índice más importante del proceso de incubación. Es de vital importancia para todo el desarrollo la reproducción en las aves, sí se tiene en cuenta que brinda información, no sólo de la cantidad de animales que se obtienen, sino también, de su calidad, pues como se sabe, se calcula a partir de los pollos de primera que se obtienen en todo el proceso reproductivo. Hoy se utiliza un nuevo índice para evaluar la reproducción, que es la cantidad de pienso consumido/pollitos de primera obtenida en la incubación; este es un parámetro que engloba la planta de incubación con el indicador más caro de la granja reproductora (Pérez 2003).

Es importante destacar que, para incubar, el huevo no sólo debe ser fértil, además debe estar apto, o sea, con las características necesarias, para dar lugar a un nuevo animal igual a la raza que le dio origen. Sin embargo, estos aspectos no han sido estudiados suficientemente, pues hay huevos que se desechan y pueden ser incubables (Pérez 2003). 
Desde lo planteado, la investigación tiene por objetivo analizar que los huevos clasificados como "no aptos" (por su peso y forma), procedentes de reproductoras pesadas, pueden ser utilizados en la incubación artificial, obteniendo de ellos, pollitos aceptables para la cría de ceba.

\section{METODOLOGÍA}

\section{Localización y duración del experimento}

La recolección de huevos se generó en las haciendas : "AMALIA", "EL GUAYABAL" y "FLOR CANELA", ubicadas en la parroquia Madre Tierra, cantón Mera, provincia de Pastaza. El proceso de incubación en la planta "INCUPASTAZA", ubicada en la vía ShellMadre Tierra, km 4, parroquia Madre Tierra, cantón Mera, provincia de Pastaza, Ecuador.

\section{Condiciones meteorológicas}

- Temperatura promedio: $21.5 \stackrel{\circ}{\circ}$

- Humedad relativa: $87.83 \%$

- Precipitación: 4.321,8 mm por año

- Altitud: 958 m.s.n.m.

\section{Materiales, Equipos e insumos.}

\section{Materiales}

Los materiales utilizados en la investigación son los siguientes:
a) Huevos "aptos" (normales)
b) Huevos "no aptos" (pequeños, grandes y deformes).
c) Flexómetro.
d) Pie de rey.
e) Overol.
f) Guantes. 

g) Cámara fotográfica.
h) Franelas y fundas.
i) Libreta de apuntes.
j) Materiales de oficina.

\section{Equipos}

Los equipos utilizados en la presente investigación son los siguientes:
a) Máquinas incubadoras.
b) Ovoscopio.
c) Pistolas de vacunación.
d) Campanas criadoras.
e) Balanza digital.
f) Computador personal.

\section{Insumos}

Los insumos utilizados en la investigación son los siguientes:

Desinfectante: Amonio cuaternario, formol.

Biológicos: Mareck, Bronquitis.

\section{Factores de estudio}

Se evaluaron 1200 huevos divididos en cuatro grupos, los mismos que fueron: 300 huevos pequeños, 300 huevos grandes, 300 huevos deformes y 300 huevos normales (aptos), procedentes de reproductoras pesadas.

\section{Tratamientos}

Consiste en evaluar cuatro grupos de huevos, procedentes de reproductoras pesadas.

a) Tratamiento 1: Testigo = Huevos "aptos" (normales). 
b) Tratamiento 2: Huevos "no aptos" (pequeños).

c) Tratamiento 3: Huevos "no aptos" (grandes).

d) Tratamiento 4: Huevos "no aptos" (deformes)

\section{Diseño experimental}

Se utilizó un Diseño de Bloques al Azar, con 3 repeticiones, conformadas por 100 unidades únicas experimentales.

\section{Análisis estadístico}

El modelo estadístico para este diseño es:

$$
y_{i j}=\mu+\tau_{i}+\beta_{j}+\epsilon_{i j}
$$

Dónde:

$\mu=$ Media general

$T i=$ Efecto generado por tratamientos

$\boldsymbol{\beta} \mathbf{j}=$ Efecto generado por bloque

Eij = Efecto del error experimental

Se aplicó ANOVA.

El análisis se complementó con la prueba de significación de TUKEY al 5\%.

Fórmula Matemática

$$
\begin{aligned}
\text { Tukey } & =(S x)\left(q_{n}\right) \\
\overline{S x} & =\sqrt{\frac{C M_{E}}{n}}
\end{aligned}
$$

\section{Mediciones experimentales}

Las mediciones experimentales que se consideraron fueron las siguientes:

- Peso del huevo, g.- El peso del huevo se determinó al momento de la recolección, 
colocándolo en la balanza digital previamente encerada, estableciéndose su peso a través de la lectura del peso en su pantalla.

- Viabilidad, \%.- La viabilidad representa la cantidad de pollitos nacidos vivos de los huevos que fueron establecidos como fértiles, y expresados en porcentaje, determinándose a través de la siguiente fórmula:

$$
\text { Viabilidad, } \%=\frac{\mathrm{N}^{0} \text { de pollitos nacidos vivos }}{\mathrm{N}^{0} \text { de huevos fértiles }} \times 100
$$

- Incubabilidad, \%.- La incubabilidad hace referencia al éxito del proceso de incubación que representa la capacidad del huevo para eclosionar, produciendo un pollo viable y se determinó mediante el siguiente enunciado matemático:

$$
\text { Índice de incubabilidad, } \%=\frac{\mathrm{N}^{0} \text { de pollos viables }}{\mathrm{N}^{0} \text { de huevos fértiles }} \times 100
$$

- ECLOSIONALIDAD = \# de pollitos nacidos / total de huevos colocados $\times 100$

- Mortalidad de los pollitos al primer día de nacidos.- La mortalidad se estableció en base al número de pollitos que se murieron después de haber nacido vivos y que aún no salían de la incubadora, determinándose mediante la siguiente fórmula:

$$
\text { Mortalidad, } \%=\frac{\mathrm{N}^{0} \text { de pollitos muertos }}{\mathrm{N}^{0} \text { de pollitos nacidos vivos }} \times 100
$$

\section{Manejo del experimento}

Las actividades que se realizaron en el desarrollo de la presente investigación se indican a continuación: 


\section{Recolección y transporte de los huevos}

Los huevos "no aptos" (por su peso y forma) y los normales utilizados para la evaluación, se recolectaron en las granjas: "AMALIA", "EL GUAYABAL" y "FLOR CANELA", cuya ubicación se mencionó anteriormente, para luego ser limpiados de las impurezas físicas de su cascara, de tal manera que exista una mejor oxigenación y óptimo desarrollo de embriones. Seguidamente se colocaron en las cubetas para ser transportados a la planta de incubación "INCUPASTAZA", ubicada en la parroquia Madre Tierra, cantón Mera, provincia de Pastaza.

\section{Proceso de incubación}

Antes de ingresar los huevos a la incubadora, estos fueron limpiados y desinfectados con amonio cuaternario, luego almacenados a una temperatura de 12 a $15^{\circ} \mathrm{C}$ con la cámara de aire hacia arriba hasta ser incubados. (Manual de procedimiento interno, INCUPASTAZA 2.012)

Una vez que la incubadora estuvo desinfectada con amonio cuaternario y precalentada, se procedió a la carga de los huevos, que se ubicaron en posición vertical, de tal manera que la cámara de aire quede en la posición más elevada, los cuales se incubaron durante 21 días. Se realizaron controles bilógicos a los 11 días mediante la ovoscopía y a los 21 días, obteniendo el peso del pollito al primer día de nacido. La temperatura de incubación fue de 37.5 a $37.7{ }^{\circ} \mathrm{C}$, con una humedad relativa promedio de $60 \%$. El volteo de los huevos durante la incubación se realizó cada hora, girando los huevos unos $45^{\circ}$, acción que se efectuó para evitar que el embrión quede adherido a las membranas y para mejorar la distribución del calor. Esta acción no se realizó nunca más después del día 18. 


\section{Traslado a las nacedoras}

El traslado a las nacedoras se lo realizó el día 19, donde permanecieron hasta el día 21, una vez nacidos los pollitos fueron clasificados en el área destinada para esto. (Sala de clasificación.).

\section{Análisis económico}

\section{Detalle}

Huevos aptos

Huevos no aptos

Ovoscopio

Pie de rey

Overol

Guantes médicos de exploración caja/100unidades

Materiales de oficina

Pistolas de Vacunación

Balanza digital

Transporte

Extras

TOTAL

\begin{tabular}{rrr} 
Cantidad & \multicolumn{2}{l}{$\begin{array}{l}\text { Precio } \\
\text { Unitario }\end{array}$} \\
300 & 0.30 & 90,00 \\
900 & 0.30 & 270,00 \\
1 & 50.00 & 50,00 \\
1 & 10,00 & 10,00 \\
2 & 20,00 & 40,00 \\
2 & 8,00 & 16,00 \\
& & 40,00 \\
2 & 45,00 & 90,00 \\
1 & 50 & 50,00 \\
& 100 & 100,00 \\
& 100 & 100,00 \\
& & 856,00
\end{tabular}




\section{CIENCIAMATRIA}

Revista Interdisciplinaria de Humanidades, Educación, Ciencia y Tecnología

Año VII. Vol. VII. N¹. Edición Especial. 2021

Hecho el depósito de ley: pp201602FA4721

ISSN-L: 2542-3029; ISSN: 2610-802X

Universidad Nacional Experimental Francisco de Miranda (UNEFM). Santa Ana de Coro. Venezuela

Diego Armando Masaquiza-Moposita; Junior Stalin Vargas-Hidalgo; Nelson Rene Ortiz-Naveda

\section{RESULTADOS Y DISCUSIÓN}

Se presentan los resultados de la investigación:

\section{Tabla 1.}

Valores medios de viabilidad por tratamiento y significación. (Letras iguales por columna no difieren significativamente).

\begin{tabular}{|c|c|c|c|c|}
\hline \multicolumn{5}{|l|}{ VIABILIDAD \% } \\
\hline Tratam/Repet & T1 & $\mathrm{T} 2$ & T3 & T4 \\
\hline R1 & 92.55 & 81.52 & 88.63 & 84.44 \\
\hline R2 & 92.7 & 82.02 & 77.01 & 88.04 \\
\hline R3 & 87.91 & 86.04 & 85.39 & 81.31 \\
\hline Tukey 5\% & A & a & $a$ & $a$ \\
\hline
\end{tabular}

Con relación al porcentaje de viabilidad, que representa la cantidad de pollitos nacidos vivos de los huevos que fueron establecidos como fértiles, se determinó que a pesar de presentar valores medios diferentes (Tabla 1 ) entre los tratamientos y repeticiones, estadísticamente no presentan diferencias de acuerdo a la prueba de Tukey al 5\%. Los valores de viabilidad encontrados en el presente trabajo están por encima de los encontrados por Juárez, (2006) quien al evaluar la viabilidad de huevos de reproductoras pesadas observó un porcentaje de viabilidad que osciló entre el 82 y $87 \%$.

Por otra parte, los valores de esta investigación también son superiores a los mencionados por Moreno et al. (2006), quien plantea que la viabilidad en ponedoras debe estar en un $80 \%$ para calificarla como buena. Sin embargo, Rosales (2010) observó una viabilidad de 92,6\% en gallinas reproductoras pesadas, al igual que Medina (2012) quien 


\section{CIENCIAMATRIA}

Revista Interdisciplinaria de Humanidades, Educación, Ciencia y Tecnología

Año VII. Vol. VII. N¹. Edición Especial. 2021

Hecho el depósito de ley: pp201602FA4721

ISSN-L: 2542-3029; ISSN: 2610-802X

Universidad Nacional Experimental Francisco de Miranda (UNEFM). Santa Ana de Coro. Venezuela

Diego Armando Masaquiza-Moposita; Junior Stalin Vargas-Hidalgo; Nelson Rene Ortiz-Naveda

encontró una viabilidad de $94,20 \%$ en investigaciones hechas en parámetros de incubación de reproductoras pesadas.

\section{Tabla 2.}

Valores medios de incubabilidad por tratamientos. (Letras iguales por columna no difieren significativamente).

\begin{tabular}{|l|l|l|l|l|}
\hline \multicolumn{6}{|l|}{ INCUBABILIDAD \% } & T1 & T2 & T3 & T4 \\
\hline Tratam/Repet & 90.42 & 76.08 & 88.63 & 81.11 \\
\hline R1 & 90.62 & 78.65 & 74.71 & 86.95 \\
\hline R2 & 86.81 & 83.72 & 82.02 & 79.12 \\
\hline R3 & a & a & a & a \\
\hline Tukey 5\% & & & & \\
\hline
\end{tabular}

La incubabilidad hace referencia al éxito del proceso de incubación o lo que es lo mismo, la capacidad del huevo para eclosionar, produciendo un pollo viable (Andrade, 2011). Según Rosales (2010), en razas de ponedoras pesadas informa un índice de incubabilidad que oscila entre 72 y $73 \%$. Los resultados de esta investigación (Tabla 5) son superiores en todos los tratamientos, siendo el de mejor comportamiento el tratamiento 1, es decir, el testigo. Los valores encontrados en los T1, T3 y T4 coinciden con los informados por Vásquez et al (2006) quienes al estudiar este indicador en huevos procedentes de reproductoras pesadas obtuvieron valores del 85 al $87 \%$.

Al igual que Fernández et al. (2004), quien observo parámetros de incubabilidad de $88,74 \%$. En el caso del tratamiento 2, los valores hallados son inferiores, lo cual pudiera establecer una relación entre el tamaño del huevo y la incubabilidad, sin embrago, Pérez (2003) obtuvo en reproductoras pesadas 62,75 y $69,8 \%$. Lesson (2004) informa valores que oscilan entre 81,1 y $86,8 \%$. 


\section{CIENCIAMATRIA}

Revista Interdisciplinaria de Humanidades, Educación, Ciencia y Tecnología

Año VII. Vol. VII. N¹. Edición Especial. 2021

Hecho el depósito de ley: pp201602FA4721

ISSN-L: 2542-3029; ISSN: 2610-802X

Universidad Nacional Experimental Francisco de Miranda (UNEFM). Santa Ana de Coro. Venezuela

Diego Armando Masaquiza-Moposita; Junior Stalin Vargas-Hidalgo; Nelson Rene Ortiz-Naveda

Los resultados obtenidos indican que los valores se hallan dentro del rango que reportan los autores antes señalados para reproductoras pesadas, pudiendo expresar que los resultados alcanzados pueden ser considerados como buenos. Como aspecto particular encontramos que el tamaño de las bandejas no era el adecuado para admitir los huevos grandes por cuanto estos tuvieron tendencia a salirse de su compartimiento por ser los mismos para huevos de menores dimensiones.

\section{Eclosionalidad}

Como se observa en la tabla 3, no hay diferencia significativa entre los tratamientos. A pesar de presentar valores medios diferentes estadísticamente no presentan diferencias de acuerdo a la prueba de Tukey al $5 \%$.

\section{Tabla 3.}

Valores medios de Eclosionalidad por tratamientos. (Letras desiguales por columnas difieren significativamente).

\begin{tabular}{|l|l|l|l|l|}
\hline \multicolumn{6}{|l|}{ ECLOSIONALIDAD \% } & T1 & T2 & T3 & T4 \\
\hline Tratam/Repet & 87 & 75 & 78 & 76 \\
\hline R1 & 89 & 73 & 67 & 81 \\
\hline R2 & 80 & 74 & 76 & 74 \\
\hline R3 & a & a & a & a \\
\hline Tukey 5\% & & & & \multicolumn{2}{l|}{} \\
\hline
\end{tabular}

Los mejores \% de eclosionalidad, se obtuvieron en el grupo testigo T1, el cual coincide con los reportados por Donohue (2010), quien encontró índices de eclosión en huevos de reproductoras pesadas de $84,5 \%$, en el resto de los tratamientos los valores fueron más bajos. Medina (2012) reporta índices de eclosión de 83,60\% en aves pesadas. Otros autores como Fernández et al. (2004) reportan un porcentaje de eclosión de 82,65\%. 


\section{CIENCIAMATRIA}

Revista Interdisciplinaria de Humanidades, Educación, Ciencia y Tecnología

Año VII. Vol. VII. N¹. Edición Especial. 2021

Hecho el depósito de ley: pp201602FA4721

ISSN-L: 2542-3029; ISSN: 2610-802X

Universidad Nacional Experimental Francisco de Miranda (UNEFM). Santa Ana de Coro. Venezuela

Diego Armando Masaquiza-Moposita; Junior Stalin Vargas-Hidalgo; Nelson Rene Ortiz-Naveda

Plano (2003) al estudiar 19 incubaciones alcanzó 81\% de eclosión. Según la guía de manejo Cobb (2008) el valor máximo de eclosión suele estar en $90 \%$.

\section{Tabla 4.}

Valores medios de los pesos de los pollitos al primer día de nacidos. (Letras distintas por columnas difieren significativamente).

\begin{tabular}{|l|l|l|l|l|}
\hline PESO (Promedio) & T1 & T2 & T3 & T4 \\
\hline Tratam/Repet & 45.64 & 34.91 & 54.77 & 48.92 \\
\hline R1 & 45.68 & 35.06 & 54.04 & 48.29 \\
\hline R2 & 46.40 & 35.46 & 52.72 & 48.74 \\
\hline R3 & c & d & a & b \\
\hline Tukey 5\% & & & & \multicolumn{5}{l|}{} \\
\hline
\end{tabular}

En la tabla 4 se muestran los pesos de los pollitos al primer día de nacidos. Los pesos obtenidos están por encima de los mencionados por Nápoles (2000) quien reportó medias de $34.6 \mathrm{~g}$ para los aptos y $34 \mathrm{~g}$ para los no aptos. Guerra y Cabrera (2003) refieren en trabajos realizados en huevos cuyos pesos eran de $49.65 \mathrm{~g}$, haber obtenido pollitos con un peso de $32.82 \mathrm{~g}$ al primer día de nacidos. Plano (2003) indica que, en huevos de peso entre 48 y $50 \mathrm{~g}$, se obtuvieron pesos al nacer de $34.6 \mathrm{~g}$.

La industria acepta un peso mínimo del huevo, requerido para una incubación exitosa, de $52 \mathrm{gr}$; esto, con la finalidad de asegurar una buena calidad del pollo cuando el principal rasgo de calidad a evaluar sea el peso (Ralph 2004). Existe una alta correlación positiva entre el peso del huevo y peso del pollo al nacer. La relación del peso del pollo y el peso del huevo se mantiene constante en la mayoría de las especies aviares. El peso del pollo esta normalmente en el rango del 62 al $76 \%$ del peso inicial del huevo, de igual forma se puede establecer esta relación en las demás especies aviares productivas. (Bruzual et al. 2000) 
En nuestras condiciones se considerará que pollitos de pesos promedios menores a 40 gramos no son viables. Como se puede observar en la tabla 7, los tratamientos 1 (normales), 3 (grandes) y 4 (deformes), tienen pesos superiores a los $40 \mathrm{~g}$, por lo que son considerados como pollitos viables, siendo los pollitos procedentes de los huevos grandes los que obtuvieron los mayores pesos. Sin embargo, coincidiendo con los planteamientos anteriores los pollitos de huevos pequeños no llegan a alcanzar el peso requerido, con un peso promedio de $35,14 \mathrm{~g}$, por lo que se consideran pollos no viables y también como pollitos de segunda.

\section{CONCLUSIONES}

Se evidenció que los huevos grandes y deformes se pueden utilizar en la incubación artificial.

Se observó que se obtuvieron pollitos de primera a partir de huevos grandes y deformes. Se comprobó que los huevos pequeños producen pollitos no viables.

\section{REFERENCIAS CONSULTADAS}

Aganga, A., Tshwenyane, O, y Molefhe, L. (2003). Influence of Feed Type on Egg. Production of Tswana Laying Chicken. International Journal of Poultry. 2(4): 2562582.

Ahmad, H. y Balander, R.(2003). Alternative Feeding Regimen of Calcium Source and Phosphorus Level for Better Eggshell Quality in Commercial Layers. Journal of AppliedPoultry Research 12:509-514.

Andrade, C. (2011). Determinación de Parámetros Reproductivos de gallinas criollas para huevos verdes desde la recolección de huevos hasta su etapa inicial. Tesis para obtener el título de ingeniero zootecnista. Escuela Superior Politécnica de Chimborazo. Andrade Galarza, C.S.

Bell, D. (2002). Satisfaciendo apropiadamente las demandas alimenticias en las ponedoras de reemplazo. Revista AviculturaProfesional. USA. Vol. 20 (6): 18-22. 
Boerjan, M. (2005). Genetic progress inspires change in incubator technology. Revista World poultry. Vol. 20(5):16-17.

Bourassa, D.V.; R. J. Buhr R.J. Wilson and J.L. (2003). Elevated Egg Holding-Room Temperature of $74^{\circ} \mathrm{F}\left(23^{\circ} \mathrm{C}\right)$. Does Not Depress Hatchability or Chick Quality, Journal of Applied Poultry Research 12: 1-6. 2003.

Brake, J. y Romero, H. (2008). Nuevos paradigmas de la Incubación y la Crianza Temprana. Departamento de Ciencias Avícolas Colegio de Agricultura y Ciencias de la Vida Universidad Estatal de Carolina del Norte Raleigh, USA. NC 27695-7608. Incubación, Biblioteca Digital, Instituto de Investigaciones Avícolas, Cuba.

Brecht, A.; Aerts, J.; Janssen, K.; Chedad, A. y Berckmans, D. (2008). Egg Shell Temperature as an Indicator for Embryonic Response,Published by the American Society of agricultalan biological Engineers, St, Joseph, Michigan Avialable, www.asebe.org.

Castañeda, P.; Ávila, A. y Esquive, J. (2001), Efecto de altos niveles de hierro adicionados a la dieta de reproductoras ligeras sobre parámetros productivos e incubabilidad. Memorias del XVII Congreso Latinoamericano de Avicultura: 55, Guatemala.

Cobb Guía de Manejo de la Incubadora (2008). Disponible en: cobb-vantress.com 11. Cuca, G. M. (2004). Estudios con calcio en gallinas ponedoras. Disponible en: http://www.Engormix.com/nuevo/prueba/colaboradoresformulariocontacto.asp?.

De Marchi, G.; Chiozzi, G.; Fasola, M. (2008). «Solar incubation cuts down parental care in a burrow nesting tropical shorebird, the crab plover Dromasardeola». Journal of AvianBiology 39 (5): pp. 484-486.

Días. P. (2014). Incubación de huevos aptos y no aptos (por su peso y forma) procedentes de reproductoras pesadas, parroquia Madre Tierra, cantón Mera, provincia de Pastaza." Tesis de Grado. Escuela de Ingeniería Agropecuaria, Universidad Estatal Amazónica. Pastaza, Ecuador. p 20.

Donohue, M. (2010). 20 años de mejoramiento avícola: Pollo de Engorde. Disponible en: http://www.elsitioavicola.com/articles/2220/20-aaos-de-mejoramiento-avacolapollo-de-engorde 
Fairchild, B. (2003). Minimización de la mortalidad embrionaria. Rev. IndustriaAvícola, Ed, Watt Publishing Co, llinois. EUA, ISSN:0019-7467. Ed. Latinoamericana de Poultry Internacional Vol. 70 (3):52, USA.

Fernández, R.; Revidatti, F.; Rafart, J.; Terraes, J.; Sandoval, Gladys.; Asiaín, M. y Sindik, M. (2004). Parámetros productivos en reproductoras de huevos y carne tipo INTA. Facultad de Ciencias Veterinarias. Universidad Nacional del Nordeste. Argentina.

Godínez, O. (2001). Curso de Postgrado de reproductores pesados y sus reemplazos (mimeo). Camagüey, Cuba.

Guerra, L. (1998). Estudio Zootécnico-Económico de las reproductoras avícolas y planta de incubación en los años 96-97. Tesis para opción al Título de Master en Producción Avícola Sostenible, Universidad de Camaguey. Camaguey, Cuba. 1020p.

Guerra, L. (2006). Potencial Productivo de los Huevos de gallina Reproductoras White Leghorn clasificados como no aptos por su peso y forma, Tesis en opción al grado de Doctor en Ciencias Veterinarias, Camaguey, Cuba.

Guerra, L. y Cabrera, L. (2003). Las pérdidas de huevos por concepto de no aptos (deformes, pequeños, grandes y rugosos) y su aporte económico en las reproductoras pesadas. Revista de Producción Animal. No 1.

Hartmann, C.; Johansson, K.; Strandberg, E. y Rydhmer, L. (2003). Genetic Correlations Between the Maternal Genetic Effect on Chick Weight and the Direct Genetic Effects on Egg Composition Traits in a White Leghorn Line. 82:1-8. PoultryScience.

John, B. P. (2000). Prevención de contaminación bacteriana en huevos, Revista de Avicultura Profesional. 18(1):22-25.

Juárez, C. (2006). Fertilidad e incubabilidad de huevos de gallinas criollas diferenciados por fenotipo en condiciones controladas. Tesis de Licenciatura. Instituto Tecnológico Agropecuario No.

Lacy, P. M. (2000). Innovación en infraestructura para broilers. Revista Avicultura Profesional. 18(2):11-12. USA. 
Langhout, D.J. (2003). El rol de los factores nutricionales en la calidad de la cáscara de huevo. En: Mesas redondas nutrición. Memorias del XVIII Congreso Latinoamericano de Avicultura: 423. Santa Cruz, Bolivia.

Lesson, S. (2004). How does breeder feed allocation affect the performance of breeders and their progeny. Revista Poultry International. 43(5): 100. USA.

Lim, H., Namkung, H. y Paik, I. (2003). Effects of Phytase Supplementation on the Performance. Egg Quality, and Phosphorous Excretion of Laying Hens Fed. 30. Madrazo, G. (2001). Curso de Postgrado sobre alimentación de las reproductoras y sus reemplazos (mimeo). Camagüey, Cuba.

Mauldin, J. (2001). Guía de análisis de huevos no eclosionados para planta de incubación. Revista Avicultura Profesional. Vol. 19 (10):18. USA.

McDaniel, G. (2002). Manejando los reproductores broilers para obtener máxima fertilidad. Revista Avicultura Profesional. Vol.20 (6):16-18. USA.

McLoughlin, G. (2000). Efecto del tamaño del huevo en el crecimiento de pollitos de engorde. Revista Avicultura Profesional. Vol. 18 (2):24.

Medina, J. (2012). Problemas del embrión de alta conformación y su incubación en sistemas de etapa única: manejo de incubadoras. XXII Congreso Centroamericano y del Caribe de Avicultura en Panamá. Panamá.

Meijerhof, R. (2004). La incubación por temperatura embrionaria. Revista Avicultura Profesional. Vol. 22 (3):13-17. USA.

Minagri (2004). Resultados de la producción de huevos en el mes de Junio del 2004 y acumulado del año, (mimeo). No 31: 8. La Habana. Cuba.

Monira, K., Salahuddin, M. y Miah, G. (2003). Effect of Breed and Holding Period on Egg Quality Characteristics of Chicken. International Journal of PoultryScience. 2 (4): 261-263.

Moreno, J., Lobato, J.; Morales, S., Merino, G., Tomas, J., Martínez, J..Sanz, R. y Soler, J. (2006). Experimental evidence that egg color indicates female condition at laying in a songbird. BehavioralEcology 17:651-655. 
Nápoles, M. (2000). Informe Científico Investigativo. Salud y Explotación de las Aves. Universidad de Camaguey. Cuba.

Narushin, V. (2001). Shape geometry of the avian egg, J, agric, Engng Res, 79(4), 441448, available online at http: www.idealibary.com

Narushin, V. y Romanov, M. (2002). Physical Characteristics of Chicken Egg in Relation to Their Hatchability and Chick Weight Available online at http:www.asabe.org

Narushin, V. y Romanov, M. (2008). Egg Physical characteristics and hatchability. Person Communication. E-mail: romanoff@pilot.msu.edu

Narushin, W. (2005). Egg Geometric Using Measurements of Lenght and breadth. Comunicasíón personal. E-mail: narushi@yahoo.com

Nilipour, H. (2000). Sistemas modernos de ventilación para broilers. Revista Avicultura Profesional. Vol. 18 (4):42. USA.

Oaxaca. p 72. 25. Keshavarz, K. (2003). Effects of Reducing Dietary Protein, Methionine, Choline, Folic Acid, and Vitamin B12 During the Late Stages of the Egg Production Cycle on Performance and Eggshell Quality. Poultry Science. 82:1407-1414.

Ortega, R. M. y Requejo, A. M. (2000). Introducción a la Nutrición Clínica. En: Nutriguía. Manual de Nutrición Clínica en Atención Primaria. Capítulo 9: 85-93. Editorial Complutense, Madrid. España.

Pérez, M. (2003). Curso de postgrado sobre Reproductores ligeros y sus reemplazos (mimeo). La Habana, Cuba.

Plano, A. (2003). Embriodiagnóstico como herramienta de trabajo para evaluar problemas de plantas de incubación y granjas reproductoras. En: Temas de producción 1.77. Memorias del XVIII Congreso Latinoamericano de Avicultura: 423. Santa Cruz de la Sierra, Bolivia.

Posadas, E.; Sánchez, E.; Rosas, C.; Ávila, E.; Téllez, I. y Quintana, J. (2001). Efecto de dos sistemas de producción sobre la calidad externa e interna del huevo en gallinas ligeras. Memorias del XVII Congreso Latinoamericano de Avicultura: 69. Guatemala.

Ralph, E. (2004). Hatching-egg production, storage and sanitation Animal. Science Department, University of California, Davis, CA 95616. USA. 
Ricks, C., Mendu, N. y Phelps, P. (2003). The Embryonated Egg: A Practical Target for Genetic Based Advances to Improve Poultry Production. PoultryScience. 82:931938. 50 .

Rosales, P. R. (2010). Evaluación Productiva de dos lotes de Gallinas Reproductoras Pesadas. Tesis Previa a la Obtención del Título de Médico Veterinario Zootecnista. Facultad de Ciencias Veterinarias. Carrera de Medicina Veterinaria y Zootecnia de la UAGRM. Santa Cruz, Bolivia.

Salazar, A. (2000). Proceso de incubación. Revista Avicultura profesional. 18(4):26. USA.

Salazar, A. (2008). Chick Master Incubator, Co. (Incubación Avícola). Nuevos conceptos, operación nacedoras. Seminario Internacional. Bogotá, Colombia.

Sardá, R. (2001). Calidad de los huevos para la incubación, curso de postgrado (mimeo). Instituto de Investigaciones Avícolas. La Habana, Cuba.

Sardá, R. (2003). Calidad de los huevos para la incubación, curso de postgrado (mimeo). Instituto de Investigaciones Avícolas. La Habana, Cuba.

Smith, T.W. (2013). Procedimiento para la incubación de huevos. Revista Los Avicultores y su Entorno, edición $N^{\circ} 94$.

Solano, Y. (2001). Informe científico - técnico para el examen estatal de Salud y Explotación Avícola. Estudio de la incubación de huevos clasificados como no aptos procedentes de reproductoras ligeras. Universidadde Camagüey. 41p. Cuba.

Summers, J. (2004). El increíble huevo. Revista Avicultura profesional. 22(1):24-26. USA. 58. UECAN, Instituto de Investigaciones Avícolas.

UECAN. (2003). Instituto de Investigaciones Avícolas. Minagri. Instructivo Técnico de Tecnología de Crianza y Regulaciones Sanitarias Generales de Reproductores ligeros y sus Reemplazos (mimeo). Cuba.

Urrutia, S. (2000). El Siglo de América Latina. Revista de Avicultura Profesional. 18(1):4. 


\section{CIENCIAMATRIA}

Revista Interdisciplinaria de Humanidades, Educación, Ciencia y Tecnología

Año VII. Vol. VII. N¹. Edición Especial. 2021

Hecho el depósito de ley: pp201602FA4721

ISSN-L: 2542-3029; ISSN: 2610-802X

Universidad Nacional Experimental Francisco de Miranda (UNEFM). Santa Ana de Coro. Venezuela

Diego Armando Masaquiza-Moposita; Junior Stalin Vargas-Hidalgo; Nelson Rene Ortiz-Naveda

Vásquez, D.; Martínez, G. y Monterrubio, R. (2006). Efecto de tres dietas en la producción, fertilidad e incubabilidad de huevos criollos y comerciales. Tesis de Licenciatura, Instituto Tecnológico Agropecuario de Oaxaca. №. 23. Nazareno, Xoxoclotlan, Oaxaca. p.72.

C2021 por los autores. Este artículo es de acceso abierto y distribuido según los términos y condiciones de la licencia Creative Commons Atribución-NoComercial-Compartirlgual 4.0 Internacional (CC BY-NC-SA 4.0) (https://creativecommons.org/licenses/by-nc-sa/4.0/). 